\title{
Análisis y Origen de las Concentraciones de TSP y PM10 en Minería de Carbón a Cielo Abierto usando Gráficos Polares
}

\author{
Roberto E. Rojano(1,2), Heli A. Arregoces ${ }^{(1,2)}$, Luis C. Angulo(1,2) y Gloria M. Restrepo(1) \\ (1) Universidad de La Guajira, facultad de ingeniería, grupo de investigación, GISA, Km 5 vía a Maicao, \\ Riohacha, Colombia. (e-mail: rrojano@uniguajira.edu.co) \\ (2) Universidad de Antioquia, facultad de ingeniería, departamento de ingeniería química, grupo PFA. Calle \\ 70 No. 52-21, Medellín, Colombia.
}

Recibido Feb. 20, 2018; Aceptado Abr. 26, 2018; Versión final May. 11, 2018, Publicado Dic. 2018

\section{Resumen}

Se estudiaron la variabilidad espacial y temporal de TSP y PM $\mathrm{PM}_{10}$ durante el período comprendido entre 2012 y 2016 en una de las minas de carbón a cielo abierto más grandes del mundo (El Cerrejón) ubicada en el

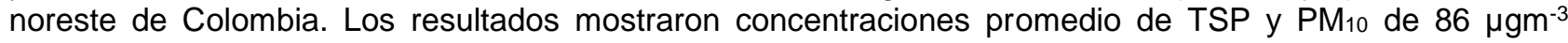
$\left(\mathrm{Cl}_{95 \%} 84-88 \mathrm{\mu gm}^{-3}\right)$ y $34{\mu \mathrm{gm}^{-3}}\left(\mathrm{Cl}_{95 \%}\right.$ 33-35 $\left.\mathrm{\mu gm}^{-3}\right)$, respectivamente. Se observó que las condiciones meteorológicas asociadas con los valores máximos de TSP y PM10, fueron vientos con velocidad mayor a 2 $\mathrm{ms}^{-1}$ y con dirección predominante NNE - NEE. Las gráficas polares y el análisis de función de probabilidad condicional estimado con el paquete openair de programa estadístico $\mathrm{R}$, mostraron que los aportes de material particulado TSP y $\mathrm{PM}_{10}$, se relaciona directamente, con las operaciones mineras desarrolladas en los pit de explotación, aunque se registran aportes significativos de $\mathrm{PM}_{10}$ de fuentes regionales, no asociadas a la explotación de carbón.

Palabras clave: concentración; material particulado; minería a cielo abierto; variaciones temporales y espaciales; gráficos polares

\section{Analysis and Origin of TSP and $\mathrm{PM}_{10}$ Concentrations in Open- pit Coal Mining using Polar Plots}

\begin{abstract}
The spatial and temporal variability of TSP and $\mathrm{PM}_{10}$ were studied during the period from 2012 to 2016 in one of the largest opencast coal mines in the world (El Cerrejón) located in northeastern Colombia. The results showed average concentrations of TSP and $\mathrm{PM}_{10}$ of $86 \mu^{-3} \mathrm{gm}^{-3}\left(\mathrm{Cl}_{95 \%} 84-88 \mu \mathrm{gm}-3\right)$ and $34 \mu \mathrm{gm}^{-3}$ $\left(\mathrm{Cl}_{95 \%}\right.$ 33-35 $\left.\mathrm{\mu gm}^{-3}\right)$, respectively. It was observed that the meteorological conditions associated with the maximum values of TSP and $\mathrm{PM}_{10}$, were winds with velocity greater than $2 \mathrm{~ms}^{-1}$ and predominant direction NNE - NEE. Polar plots and conditional probability function analysis showed that the contributions of particulate material TSP and $\mathrm{PM}_{10}$, is directly related to the mining operations developed in the pit, although there are significant contributions of $\mathrm{PM}_{10}$ from regional sources not associated with the open-pit coal mine.
\end{abstract}

Keywords: concentration; particulate matter; open pit mining; temporal and spatial variations; polar plots 


\section{INTRODUCCIÓN}

El carbón en la actualidad suministra el $25 \%$ de la energía primaria consumida en el mundo, solo por detrás del petróleo. Además, es de las primeras fuentes de energía eléctrica, con el $40 \%$ de producción mundial. Algunos de sus más importantes usos corresponden a la generación de electricidad, la producción de acero y la fabricación de cemento. Colombia es el primer productor de carbón de América Latina y el octavo del mundo (BP, 2016). Es el único país latinoamericano en los veinte primeros productores. Sus reservas medidas son de 7.063,6 Mt, ubicadas principalmente en la Costa Atlántica, norte de Colombia, donde se ubica el departamento de La Guajira.

En el departamento de La Guajira se genera aproximadamente el 39\% de la producción nacional de carbón y es donde se ubica una de las 10 minas a cielo abierto más grandes del mundo, llamada La Mina Cerrejón (UPME, 2016). La mina el Cerrejón, tiene una reservas de $3694,91 \mathrm{Mt}$, correspondiente al $56,77 \%$ del total de la reservas de Colombia (Monroy et al., 2014). Tiene actualmente una superficie aproximada de $800 \mathrm{~km}^{2}$, con una capacidad extractiva de 30-32 millones de toneladas de carbón al año, lo que representa aproximadamente el $41 \%$ del total de las exportaciones colombianas (Cerrejón, 2011). Las grandes minas a cielo abierto como el Cerrejón, requieren que se elimine la capa de tierra, rocas y vegetación que cubre las costuras de carbón. Esto da lugar a la emisión de contaminantes y partículas a la atmósfera, que pueden producirse por las perturbaciones de la tierra por explosivos y la erosión eólica. Además, también son importantes las emisiones de la producción y procesamiento de carbón, como el polvo de transporte y el escape de diesel de los equipos de minería y transporte.

La extracción de carbón a cielo abierto involucra actividades de manipulación de material tierra o carbón. Todas las operaciones que impliquen movimientos de tierra o carbón o la exposición de las superficies erosionables, generan emisión de material particulado (USEPA, 2007). El material aerotransportado ha sido identificado como la principal fuente de contaminación del aire en la zona de influencia de las extracciones, especialmente las partículas suspendidas totales (TSP) y las partículas menores de 10 micras (PM 10$)$ (Ghose y Majee, 2000; Watson et al., 2000). La emisión de este tipo contaminante ha sido de interés en el contexto científico por el efecto nocivo a la salud. La exposición de la salud humana a los contaminantes particulados ha sido investigada extensivamente y asociada con excesos en la morbilidad y mortalidad (Brunekreef, 2002; Pope y Dockery, 2006; Shi et al., 2015). Se han realizados estudios en poblaciones con proximidad a áreas de minería de carbón a cielo abierto mostrando coincidencias entre su proximidad a la actividad industrial y elevadas tasas de mortalidad de la población (Hendryx, 2015; Hendryx y Zullig, 2009). Inclusive, un estudio reciente mostró la incidencia del cáncer de pulmón y la mortalidad con la proximidad a una mina de carbón. Sin embargo, los investigadores sugirieron más estudios a nivel de exposiciones individual entre los efectos ambientales de la minería de carbón y los resultados del cáncer (Mueller et al., 2015). De lo anterior, se observa la importancia de identificar las fuentes de emisiones de partículas locales y distantes. La literatura reporta mucha información de técnicas para identificar fuentes de contaminación. Por ejemplo, los modelos de receptor es una técnica importante en la gestión de la contaminación del aire para identificar aportes (Hopke, 2010; Watson y Chow, 2015).

Entre otras técnicas, hay un método utilizado para la identificación de las fuentes de contaminación, teniendo claro la ubicación de las estaciones de monitoreo y las condiciones meteorológicas de la zona. Este método utiliza la velocidad del viento local y los datos de dirección para identificar las direcciones posibles de ubicación de las fuentes locales. De hecho, la relación de la dirección y velocidad del viento con la concentración de los contaminantes atmosféricos y la utilización de herramientas gráficas, tales como la rosa de los vientos, la rosa de contaminantes y los gráficos polares, ayudan a identificar aportes de fuentes cercanas. Toro et al. (2014) y García y Rojas (2016), determinaron la procedencia de los contaminantes del aire registrados por una red de monitoreo como acciones de prevención y control de la contaminación de material particulado en las ciudades de Santiago y Bogotá, respectivamente. Este estudio tiene como objetivos identificar las fuentes principales de contaminación de particulas $\mathrm{PM}_{10}$ en la zona de influencia de la mina el Cerrejón usando función de probabilidad condicional. Los gráficos polares fueron utilizados ampliamente para investigar las relaciones de pares de contaminantes y como herramienta para separar fuentes y procesos contaminantes (Grange et al., 2016). Estas graficas han demostrado ser útiles para obtener rápidamente una impresión gráfica de fuentes potenciales que influyen en un lugar y usan la técnica de suavizamiento estadístico para mostrar una superficie continua (Carslaw, 2015).

\section{METODOLOGÍA}

Se presenta la metodología empleada en la investigación desarrollada en tres secciones que comprenden: descripción del lugar de estudio, la metodología de obtención de las cconcentraciones de partículas y datos meteorológicos y las herramientas utilizadas en el análisis de los dato. 


\section{Descripción del área de estudio}

El yacimiento de carbón el Cerrejón ( $11^{\circ} 5^{\prime} 2^{\prime \prime} \mathrm{N} ; 72^{\circ} 40^{\prime} 31^{\prime \prime} \mathrm{W}$, ver Figura 1 ) es una mina a cielo abierto ubicada en el departamento de La Guajira, al noreste de Colombia, muy cerca de la frontera con Venezuela, entre los municipios de Albania, Hatonuevo y Barrancas, a una distancia de $150 \mathrm{~km}$ de la costa, a $100 \mathrm{~km}$ de Riohacha, capital del departamento de La Guajira, a $290 \mathrm{~km}$ de la ciudad vacacional de Santa Marta y a $400 \mathrm{~km}$ de la ciudad industrial de Barranquilla. La población total del departamento de La Guajira no supera los 900.000 habitantes. Alrededor del yacimiento carbonífero habitan 59.089 personas, de los cuales el 34\% son indígenas. El 70\% de dicha población, se ubica a menos de $10 \mathrm{~km}$ del yacimiento. Los depósitos de carbón se localizan en un área aproximada de 80.000 hectáreas.

El carbón aparece a diferentes profundidades, buzamientos y con estructuras muy dispares como consecuencia de la geología de la zona. El techo de las capas superiores de carbón puede variar entre 10 y 50 metros, y su espesor entre varios centímetros y casi la decena de metros. La profundidad máxima a la que se está extrayendo carbón en Cerrejón es a 200 metros bajo la cota 0. La operación minera alcanzó una producción de carbón de 33,4 millones de toneladas (Mt) durante 2015. Para el 2016 bajo a 32 Mt debido a cierre de algunos tajo, por la influencia del fenómeno El Niño. La mina tiene aprobados planes de expansión para aumentar la producción a 42 Mt en 2017 (Cerrejón, 2011). La operación se desarrolla en 6 tajos de explotación denominadas, de norte a sur: La Puente pit (LPUp), Tabaco pit (TABp), Patilla pit (PATp), Comunero pit (COMp), 100 pit (100p) y Oreganal pit (OREp).

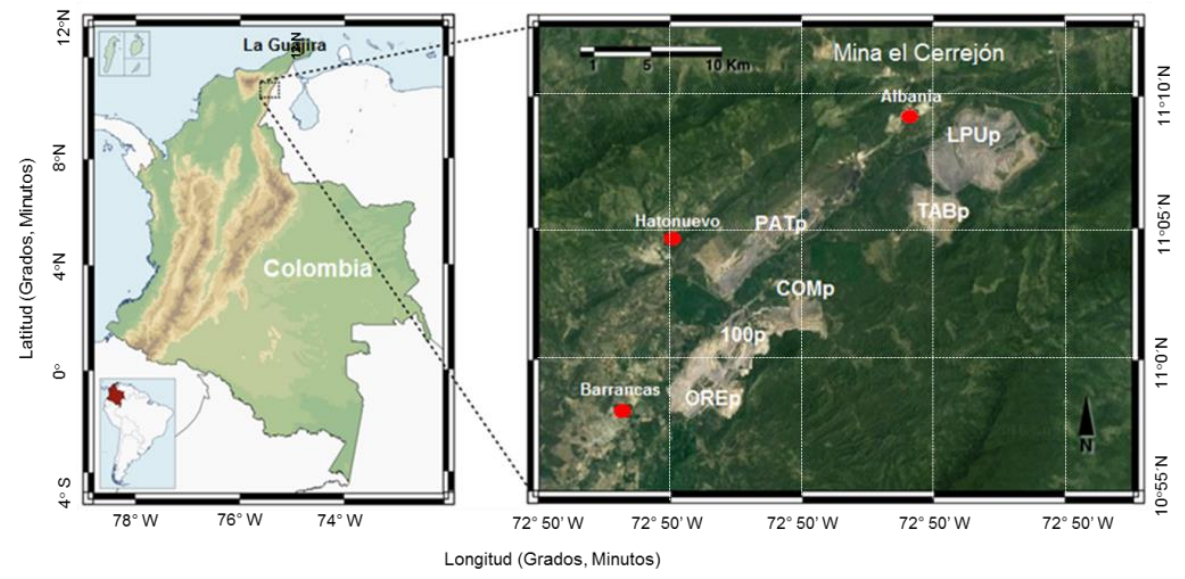

Fig. 1: Localización geográfica de la mina de carbón Cerrejón y las estaciones de monitoreo en La Guajira, región del Caribe de Colombia. Los puntos rojos son las ciudades aledañas a la mina. (Panel izquierdo: modificado de Mapa de Colombia (orografía) .svg Wikipedia, https://goo.gl/Wg6Tzl, consultado 2016-03-02; panel del rigth: fuente google tierra v7.1.5.1557, 2016-03- 02, DigitalGlobe 2016. http://www.earth.google.com, consultado el 2017-03-10).

La topografía del terreno se caracteriza por ser una llanura aluvial en la cuenca del río Ranchería, entre los sistemas montañosos de la serranía de Perijá en el este y la Sierra Nevada de Santa Marta en el oeste. El clima está fuertemente influenciado por la proximidad a los sistemas montañosos de la serranía de Perijá y Sierra Nevada de Santa Marta. La Guajira es de clima cálido catalogada como la zona más seca de Colombia. La Tabla 1 presenta una descripción de los promedios, desviaciones, valores máximos y mínimos de los parámetros meteorológicos entre enero y junio de 2012 (Rojano et al., 2016).

Tabla 1: Promedios de los parámetros meteorológicos en el periodo enero - junio de 2012.

\begin{tabular}{lccc}
\hline \multicolumn{1}{c}{ Parámetro } & Media (Desv. Est) & Máximo & Mínimo \\
\hline Temperatura $\left({ }^{\circ} \mathrm{C}\right)$ & $27,00(4,72)$ & 36,00 & 17,60 \\
Viento $(\mathrm{m} / \mathrm{s})$ & $3,31(0,70)$ & 6,04 & 2.53 \\
Humedad Relativa $(\%)$ & $80,20(7,24)$ & 85,00 & 64,00 \\
Presión barométrica $(\mathrm{mmHg})$ & $758,00(10)$ & 760,00 & 755,00 \\
Precipitación $(\mathrm{mm}$ anual) & $909,00(17,60)$ & 152,00 & 0,10 \\
Evaporación $(\mathrm{mm})$ & $5,54(1,44)$ & 9,62 & 1,31 \\
\hline
\end{tabular}

Las lluvias son escasas y mal distribuidas. Las precipitaciones se distribuyen según un pronunciado patrón bimodal, con una estación lluviosa de cuatro meses, de septiembre a diciembre. Posteriormente, se presenta una temporada de sequía de enero a abril por la presencia de los vientos alisios con fuerte intensidad predominando del noreste. A causa de un debilitamiento de los vientos se presentan 
precipitaciones y corresponde a la segunda estación húmeda en el mes de mayo. Presentándose luego, la segunda temporada de sequía entre los meses de junio a agosto ( $\mathrm{ClOH}, 2010$; Ramírez y del Valle, 2012).

\section{Concentraciones de partículas y datos meteorológicos}

Las variables meteorológicas (temperatura, precipitaciones, velocidad y dirección del viento) y las concentraciones ambientales de TSP y $\mathrm{PM}_{10}$ utilizados en el estudio, se obtuvieron de las estaciones de la Red de Calidad del Aire del Cerrejón. La Tabla 2 muestra la localización de las estaciones de monitoreo y los parámetros medidos en cada una de ellas. Las estaciones seleccionadas están localizadas en la zona norte: estación Sol y Sombra (SS); zona centro: estación Patilla (PT) y estación Provincial (PV) y zona sur: estación Barranca (BR) y estación Las Casitas (LC). Las estaciones de muestreo, su ubicación geográfica y las variables medidas, se presentan en la Figura 2. Los sitios SS y BR representan las estaciones viento arriba y viento abajo (según los vientos prevalecientes), útiles para determinar el flujo de contaminantes, dentro y fuera de la zona de estudio. Las estaciones PT, PV y LC, son representativas de las actividades extractivas desarrolladas en la mina. Adicionalmente, los sitios PV y LC, incluyen otras actividades realizadas por los habitantes ubicados en zonas adyacentes a estas estaciones, tales como: quema de biomasa, áreas destapadas y transporte en vías destapadas. Las estaciones PT y SS, no tienen sensores meteorológicos, por lo que fue necesario estimar las variables velocidad del viento, dirección del viento y temperatura. La estimación se realizaron con el modelo meteorológico CALMET del CALPUFF, usando la metodología explicada por Scire et al., (2000).

Tabla 2: Ubicación y elevación de la red meteorológica del Cerrejón utilizada en esta investigación. T: temperatura; P: precipitaciones vv: velocidad del viento; dv: dirección del viento. * Variables estimadas por modelación meteorológica.

\begin{tabular}{|c|c|c|c|c|c|}
\hline$I D$ & Estaciones & Latitud $\left(^{\circ}\right)$ & Longitud $\left({ }^{\circ}\right)$ & $\begin{array}{l}\text { Elevación } \\
\text { (m) }\end{array}$ & Variables medidas \\
\hline $\mathrm{SS}^{*}$ & Sol y Sombra & 11.143 & -72.519 & 117 & TPS, PM10, T, vv y dv \\
\hline $\mathrm{PT}^{*}$ & Patilla & 11.050 & -72.671 & 115 & TPS, PM10, T, vv y dv \\
\hline PV & Provincial & 11.022 & -72.742 & 156 & TPS, $\mathrm{PM}_{10}, \mathrm{~T}, \mathrm{P}, \mathrm{vv}$ y dv \\
\hline $\mathrm{BR}$ & Barrancas & 10.960 & -72.780 & 150 & TPS, $\mathrm{PM}_{10}, \mathrm{~T}, \mathrm{P}, \mathrm{vv}$ y dv \\
\hline LC & Las Casitas & 10.955 & -72.741 & 162 & TPS, PM10, T, P, vv y dv \\
\hline
\end{tabular}

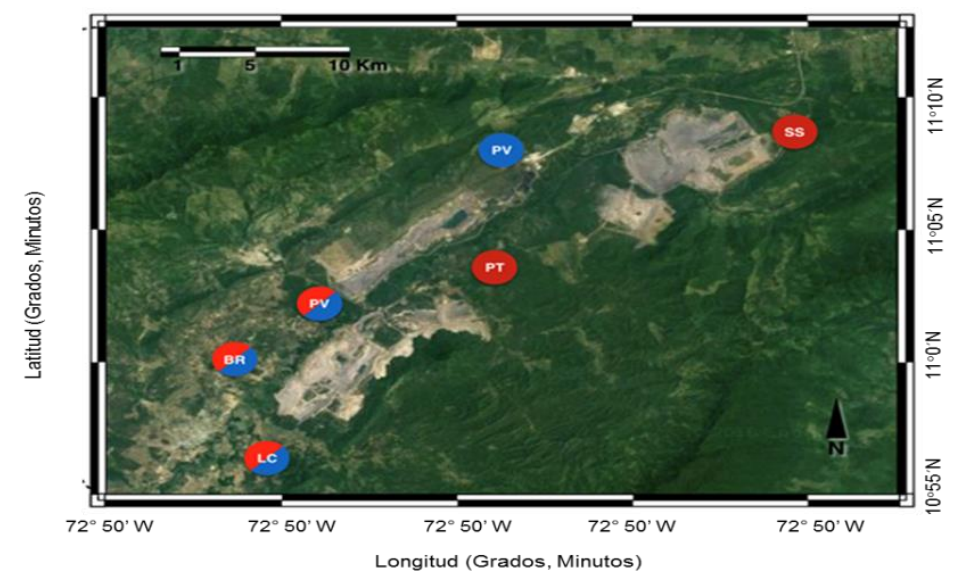

Fig. 2: Localización geográfica de la mina de carbón del Cerrejón y de las estaciones de monitoreo. ID estaciones BRes Barrancas; LC es Las Casitas; PT es Patilla; PV es Provincial y SS es Sol y sombra.

La concentración de material particulado TSP y $\mathrm{PM}_{10}$ se determinaron por el método gravimétrico con muestreador de alto volumen, procedimiento establecido en el Reference Method of the CFR (Code of Federal Regulations) (Appendix B to Part 50 for TSP and Appendix J to Part 50 for PM 10 ). Se empleó equipos Hi-Vol para TSP y PM 10 de la Marca Tisch Environmental, modelo TE-6070 para PM10 y modelo TE5170 para TSP. Se utilizaron filtros de cuarzo (Whatman tamaño de 8 por 10 pulgadas, tamaño de poro de 2 $\mu \mathrm{m})$ para el muestreo de TSP y $\mathrm{PM}_{10}$.

\section{Análisis de los datos meteorológicos y de concentraciones TSP y $\mathrm{PM}_{10}$}

Los datos de concentraciones TSP y $\mathrm{PM}_{10}$ y los datos meteorológicos, se tabularon en una hoja de cálculo 
(MS-Excel® 2016 para Windows, Microsoft Corporation, Redmond, WA, EE.UU.). El análisis estadístico descriptivo se realizó utilizando el mismo software (MS-Excel (C) 2011) con la herramienta de tablas dinámicas. Para los cálculos estadísticos más avanzados, se utilizó el software estadístico de código abierto $\mathrm{R}$ (RCoreTeam, 2016. Lenguaje y medio ambiente para la informática estadística, R Foundation for Statistical Computing, Viena, Austria) con el entorno de desarrollo integrado RStudio para R (RStudio Boston, MA. Http://www.rstudio.org/) y el paquete de herramientas para el análisis de la calidad del aire en R llamado OpenAir (Carslaw, 2015; Carslaw y Ropkins, 2012). El análisis de la rosa del viento se realizó utilizando la opción polarPlot del programa R (Carslaw y Beevers, 2013). Para el cálculo se tomaran las concentraciones diarias de $\mathrm{PM}_{10}$ en cada estación y se le asignaron su correspondiente dirección de viento diario. Como la dirección y velocidad del viento estaba en forma horaria, se hizo necesario expresar estos parámetros meteorológicos en promedios diarios. Estos promedios se calculan obteniendo los componentes del viento $(\vec{u}, \vec{v})$ y aplicando la formula citada por Grange (2014). De igual manera, el CPF para cada estación se calculó utilizando el paquete openair del software estadístico $R$, con la función percentileRose (Uria-Tellaetxe y Carslaw, 2014).

\section{RESULTADOS Y DISCUSIÓN}

Se presentan los resultados del análisis y origen de las concentraciones de TSP y $\mathrm{PM}_{10}$ en minería de carbón a cielo abierto usando gráficos polares.

\section{Meteorología}

La Tabla 3, presenta un resumen estadístico de los parámetros meteorológicos en las cinco estaciones, con datos obtenidos con sensores (PV, BR y LC) y datos estimados, con modelos meteorológicos (PT y SS). El promedio de la velocidad del viento observada en las estaciones de PV, BR y CA, fue de $3 \mathrm{~ms}^{-1}$ (Cl95\%: 1-5 $\mathrm{ms}^{-1}$ ). Los mayores promedios de velocidad del viento, se presentaron en la estación LC, con valor de 3.4 $\mathrm{ms}^{-1}$ en el año 2015. La dirección del viento predominante en las tres estaciones con sensores y en las dos estaciones con parámetros estimados, fue ENE y NE, seguida de la dirección E y NNE. En todas las estaciones se presentaron direcciones SE, con menor probabilidad, indicando arrastre de contaminantes de las poblaciones a las estaciones. La Figura 3, muestra las frecuencias de dirección de viento y variación de la velocidad del viento para todas las estaciones, periodo 2012 - 2016. Los resultados del estudio de la temperatura, registraron comportamientos similares en las estaciones PV, BR y LC, con promedios de 20,39 ${ }^{\circ} \mathrm{C}, 29,01^{\circ} \mathrm{C}$ y $29,69{ }^{\circ} \mathrm{C}$, respectivamente. La máxima temperatura se presentó en PT $\left(36,82{ }^{\circ} \mathrm{C}\right)$ y el valor mínimo se registró en $\mathrm{LC}\left(13,30^{\circ} \mathrm{C}\right)$. El promedio general para las estaciones de PV, BR y LC se centra en un valor de $29,00^{\circ} \mathrm{C}$ (Cl95\%: 27-32). La temperatura se caracterizó por ser alta, desde abril a septiembre, y los menores valores, durante la otra mitad del año, desde octubre a marzo. Las temperaturas medias anuales, por lo general, son mayores de $27,00^{\circ} \mathrm{C}$.

Tabla 3: Resumen estadístico de los parámetros meteorológicos mensuales en la mina el Cerrejón.

\begin{tabular}{crrrrrrrrr}
\hline \multicolumn{1}{c}{ Temperatura $\left({ }^{\circ} \mathrm{C}\right)$} & \multicolumn{7}{c}{ Humedad relativa (\%) } \\
\hline Estación & Promedio & StDev & Min & Max & Promedio & StDev & Min & Max \\
\hline SS & 28.22 & 2.80 & 12.80 & 34.80 & & & & \\
\hline PT & 29.82 & 2.01 & 13.25 & 36.82 & & & & \\
\hline PV & 29,23 & 1,83 & 12.80 & 34.80 & 59.15 & 10,23 & 18.00 & 96.33 \\
\hline BR & 28.89 & 1.72 & 25.13 & 33.17 & 64,75 & 10.68 & 38.66 & 92.18 \\
\hline LC & 29.69 & 1,97 & 13.25 & 36.82 & 64,01 & 10.80 & 18.00 & 96.20 \\
\hline \multicolumn{1}{c}{ Precipitación $(m m)$} & & & Velocidad del viento (m/s) \\
\hline Estación & Promedio & StDev & Min & Max & Promedio & StDev & Min & Max \\
\hline SS & 1.60 & 7.64 & 4.56 & 119.20 & 2.66 & 0.95 & 0.30 & 5.39 \\
\hline PT & 1.50 & 6.66 & 1.23 & 76.60 & 2.54 & 0.96 & 0.18 & 6.04 \\
\hline PV & 1.84 & 7,61 & 1.10 & 76.60 & 2,89 & 1.11 & 0.00 & 5.71 \\
\hline BR & 1.93 & 7,73 & 3.45 & 110.30 & 2.83 & 1.02 & 0.00 & 5.24 \\
\hline LC & 1.85 & 7.56 & 2.67 & 107.20 & 3.06 & 1.11 & 0.00 & 5.71 \\
\hline
\end{tabular}

Los períodos de mayor temperatura corresponden a las estaciones secas y los periodos de menor temperatura corresponden a las estaciones lluviosas. En general, los resultados son consistentes y aceptables para el área de estudio. Las estaciones de PV, BR y LC, presentaron promedios mensuales de precipitaciones de 2,38, 2,44 y 2,41 mm, respectivamente. Se registraron 77 días de lluvia en promedio, en 
las cinco estaciones, en el período de estudio. El año 2015 presentó el promedio más bajo, con 13 días de lluvia. El máximo registro diario se presentó en BR $(110 \mathrm{~mm})$ y el registro mínimo se registró en LC $(0,1$ $\mathrm{mm})$. El promedio general para las estaciones, de PV BR y LC, se centra en un valor de $1,89 \mathrm{~mm}$ ( $\mathrm{Cl}_{95 \%}$ : 1,69-2,10). El comportamiento bianual del máximo de lluvias en octubre y noviembre y los mínimos de enero y febrero, son asociados con la mayor actividad (intensidad) del Chorro del Chocó, al igual que para en el resto del Colombia.

No se registraron tendencias estadísticamente significativas en velocidad del viento, temperatura y precipitaciones en las estaciones PV, BR y LC, en el periodo 2012 - 2016. Sin embargo, si se presentó tendencia baja positiva en la humedad relativa, en las estaciones BR y LC. Los cambios de tendencias que se presentan en estas variables, son inducidos por la topografía y la climatología. Para este caso, son influenciados por el clima, en la relación océano - continente, al encontrarse esta zona en el Caribe colombiano. Las variables estudiadas en las tres estaciones, presentan una buena correlación. Por lo tanto, los resultados de las variables meteorológicas en el período, de estudio son representativo del clima de la zona y son coherentes, en comparación con los resultados de estudios anteriores, pero, desde una óptica nacional (Mesa et al., 2000; Pabón-Caicedo y Eslava-Ramírez, 2001).

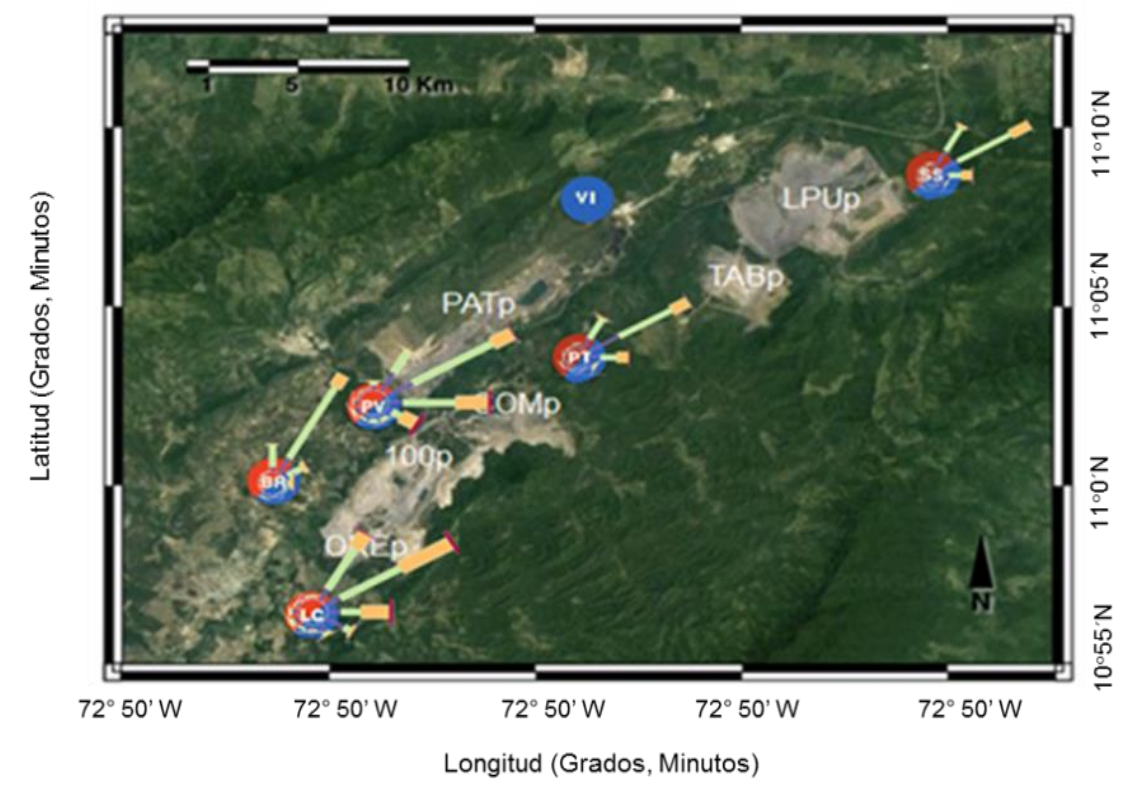

Fig. 3: Rosa de dirección de viento para cada una de las estaciones, periodo 2012 - 2016, zona minera el Cerrejón. Las columnas de la figura, se trazan en coordenadas polares

\section{Concentraciones anuales de TSP y $\mathrm{PM}_{10}$}

La Figura 4, muestra la distribución de las concentraciones TSP y $\mathrm{PM}_{10}$, en las cinco estaciones, entre el periodo 2012 a 2016. Los gráficos boxplot, muestran la mediana (línea media de la caja), el intervalo de confianza (superior e inferior del $95 \%$ ), el cuartil superior (25\%) y el inferior (75\%), la distribución de los promedios y los valores máximos y mínimos, de cada estación. Las estaciones BR, PT, PV y LC, presentan una distribución similar, observándose mayor dispersión en la estación PV y LC, para el material particulado TSP y en la estación LC para PM10. La estación SS, presenta los menores valores en la distribución, lo cual es coherente con la posición que ocupa en la geografía del área minera. Esta estación, se encuentra viento arriba de las operaciones industriales. La media fue mayor que la mediana, en todas las estaciones, lo que indica el hecho, que el conjunto de datos se sesgan a la derecha, por la ocurrencia de emisiones significativas y constantes. De igual manera, no se observa gran número de valores anómalos en los boxplots (puntos fuera de la línea de bigotes). Esto indica concentraciones de TSP y $\mathrm{PM}_{10}$ con un comportamiento constante. Este comportamiento es coherente con concentraciones de una misma fuente de emisión.

La estación LC tiene los mayores niveles de TSP y PM10, seguido de la estación PV y PT. Estas estaciones, están en el corazón de las actividades mineras. Diferente comportamiento se aprecia con los valores mínimos; esta distribución se solapa con los valores de la estación SS, indicando la presencia de fuentes de emisión diferentes, que afectan a la estación SS. Se puede apreciar la similar distribución de TSP y PM10 para la estación SS; esta distribución está en el rango de 0 a $50 \mathrm{\mu gm}^{-3}$ lo que indica que gran porcentaje del TSP corresponde a PM10, observándose que fuentes diferentes a las actividades mineras, hacen aportes a la estación SS. 
También se observa que la concentración de TSP es mayor a la de $\mathrm{PM}_{10}$ en todas las estaciones. Así mismo se aprecia que existe un ciclo anual con mayores concentraciones en el primer semestre de cada año. Es posible establecer, que en promedio, para todas las estaciones en estudio entre 2012-2016, la

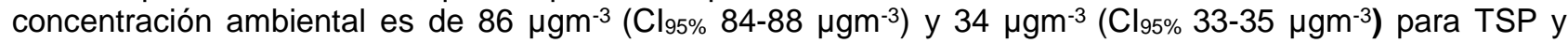
$\mathrm{PM}_{10}$, respectivamente. Se evidencia que las concentraciones de TSP son mayores a las de $\mathrm{PM}_{10}$, en 2.5 veces. Un análisis ANOVA, mostró que las concentraciones de TSP y $\mathrm{PM}_{10}$ son estadísticamente diferentes a un $95 \%$ de intervalo de confidencia ( $p$-value $<0.05$ ).

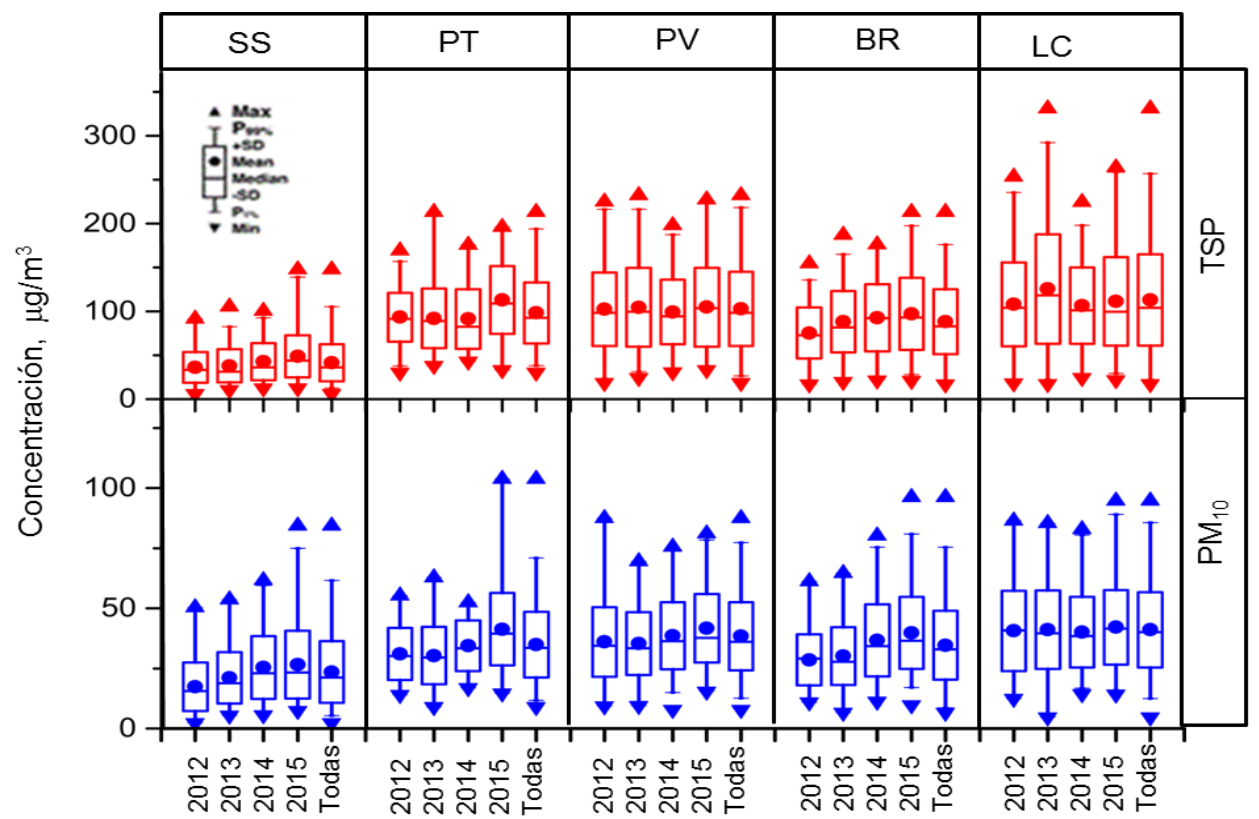

Fig. 4: Boxplot de las concentraciones TSP y $\mathrm{PM}_{10}$ en las cinco estaciones. Periodo 2012 2016. Las cajas muestran la mediana (línea media de la caja) el intervalo de confianza (superior e inferior del 95\%), el cuartil superior (25\%) y el inferior $(75 \%)$.

En el caso de PST las mayores concentraciones se registran en la estación de LC (96 $\mu$ gm $^{-3}$; Cl95\% 93-102

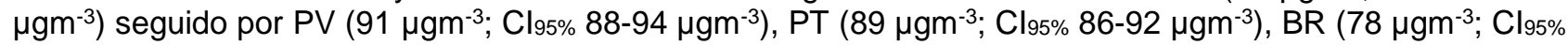
75-81 $\left.\mathrm{\mu gm}^{-3}\right)$, y las menores concentraciones, se observan en la estación SS (37 $\mathrm{ggm}^{-3}$; Cl $\left.195 \% 35-38 \mathrm{\mu gm}^{-3}\right)$. Con relación a $\mathrm{PM}_{10}$, las mayores concentraciones se observan en LC $\left(41 \mathrm{\mu gm}^{-3}\right.$; Cl ${ }_{95 \%}$ 40-42 $\left.\mathrm{\mu gm}^{-3}\right)$,

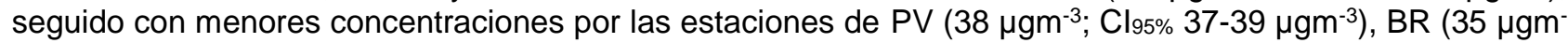

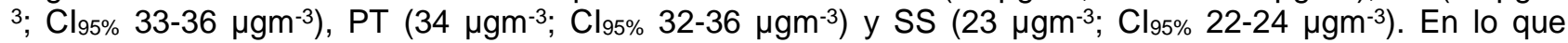
respecta a la variabilidad interanual de las concentraciones, tanto para TSP como para $\mathrm{PM}_{10}$, un análisis ANOVA mostró que no existe diferencia estadísticamente significativa a un $95 \%$ de intervalo de confidencia ( $p$-valor > 0.05). Los niveles de concentración de TSP y PM10 son parecidos a estudios realizados en la misma zona de La Guajira (Argumedo y Castillo, 2016; IDEAM, 2016) pero menores que datos de estudios TSP y PM10 registrados en otras minas de carbón ubicadas al norte de Colombia, más al sur del Cerrejón (Castro y Rojas, 2015; Rodríguez et al., 2014).

\section{Efectos de las variables meteorológicas}

La Tabla 4, muestra el coeficiente de correlación de Pearson entre TSP, PM10 y las variables meteorológicas (temperatura, humedad relativa, precipitación y velocidad del viento). Se encontró una correlación negativa entre TSP, $\mathrm{PM}_{10}$, con la humedad relativa y las precipitaciones. Los coeficientes de correlación para la humedad relativa, varían en un rango de entre $-0,044$ a $-0,323$ y $-0,088$ a $-0,210$ para TSP y $\mathrm{PM}_{10}$, respectivamente. En el caso de las precipitaciones, los coeficientes varían entre -0,191 a -0,248 y -0,138 a 0,186 para TSP y $\mathrm{PM}_{10}$, respectivamente. Para la temperatura tanto para TSP y $\mathrm{PM}_{10}$, la correlación es positiva y tiene una variación que va entre 0,162 a 0,200 y 0,152 a 0,334, respectivamente. Puede ser que estas correlaciones se deba, a los efectos de la humedad relativa/precipitaciones y de la temperatura, en la remoción por deposición húmeda por lluvia y deposición seca, obtenida por rocío.

Al analizar la correlación entre la velocidad del viento y las concentraciones de TSP y PM10, vemos que existe una correlación positiva que varía entre 0,356 a 0,620 y 0.219 a 0,453, respectivamente. La velocidad del viento es importante en el estudio de las concentraciones de partículas, ya que es el factor principal que determina el transporte y dispersión de las partículas, a las diferentes zonas. Las TSP y PM10, pueden ser 
dispersadas a mayor velocidad de vientos. Las correlaciones observadas con la temperatura, humedad relativa y velocidad del viento, con partículas atmosféricas, han sido reportadas con iguales resultados por otros investigadores (Rojano et al., 2018; Huertas et al., 2014).

Tabla 4: Análisis de correlación de Pearson entre el TSP, $\mathrm{PM}_{10}$ vs temperatura $(\mathrm{T})$, humedad relativa (HR) precipitaciones (lluvia) y velocidad de viento, para cada estación en estudio en el período comprendido entre los años 2012-2016. (BR: Barrancas, LC: Las Casitas y PV: Provincial

\begin{tabular}{lcccc}
\hline & \multicolumn{4}{c}{ Variables meteorológicas } \\
\cline { 2 - 5 } Estaciones & $\begin{array}{c}\text { Temperatura } \\
{ }^{\circ} \mathrm{C}\end{array}$ & $\begin{array}{c}\text { Humedad relativa } \\
\%\end{array}$ & $\begin{array}{c}\text { Precipitaciones } \\
\mathrm{mm}\end{array}$ & $\begin{array}{c}\text { Velocidad del viento } \\
\mathrm{m} / \mathrm{s}\end{array}$ \\
\hline BR & 0,200 & $-0,323$ & $-0,223$ & 0,62 \\
LC & 0,162 & $-0,132$ & $-0,191$ & 0,356 \\
PV & 0,173 & $-0,044$ & $-0,248$ & 0,512 \\
\hline \multicolumn{5}{c}{$P M_{10}$} \\
\hline BR & 0,334 & $-0,21$ & $-0,155$ & 0,344 \\
LC & 0,289 & $-0,168$ & $-0,138$ & 0,219 \\
PV & 0,152 & $-0,088$ & $-0,186$ & 0,453 \\
\hline
\end{tabular}

\section{Cumplimiento de la norma nacional e internacional}

La Tabla 5, contiene los promedios anuales de los datos de TSP y $\mathrm{PM}_{10}$. Estos resultados se evaluaron según la guía de WHO y La norma colombiana para promedios diarios y anuales de PM10 (MADS, 2017). Para el material particulado $\mathrm{PM}_{10}$, el análisis estadístico de los promedios, muestra que no fueron superados el nivel máximo permisible diario $\left(100 \mu \mathrm{g} / \mathrm{m}^{3}\right)$ y el nivel máximo permisible anual $\left(50 \mu \mathrm{g} / \mathrm{m}^{3}\right)$. Todos los promedios en las estaciones BR, LC, PT, PV, superan el nivel anual $\left(20 \mu \mathrm{g} / \mathrm{m}^{3}\right)$ de la guía de la Organización Mundial de la Salud (GDL-WHO), en el periodo 2012 -2016. Comparando los resultados, con el promedio diario de la OMS $\left(20 \mu \mathrm{g} / \mathrm{m}^{3}\right)$, el análisis muestra excedencia del $13 \%, 25 \%, 11 \%, 20 \%$ y el $4 \%$ en la estaciones BR, LC, PT, PV y SS, respectivamente.

Sin embargo, los niveles de $\mathrm{PM}_{10}$, registrados en la mina el Cerrejón son inferiores a niveles registrados en otras minas a cielo abierto del norte de Colombia, inclusive menores en comparación con zonas urbanas de las principales ciudades de Colombia (IDEAM, 2016). Colombia no establece niveles máximos permisibles para TSP. Sin embargo, tomando como base la Directiva 80/779, de la Agencia Europea Ambiental la Unión Europea que establece un valor límite de TSP de $150 \mu \mathrm{g} / \mathrm{m}^{3}$ anual; ninguna de las estaciones tiene promedios anual por encima de esta norma (EEA, 2008).

Tabla 5: Promedios anuales de material particulado PM10 y TSP en cada estación

\begin{tabular}{cccccc}
\hline \multicolumn{5}{c}{$T S P\left(\mu g / m^{3}\right)$} \\
\hline$A \tilde{n}$ o & $B R$ & $L C$ & $P T$ & $P V$ & $S S$ \\
\hline 2012 & 69,08 & 96,21 & 89,01 & 92,68 & 32,40 \\
2013 & 80,63 & 109,64 & 86,41 & 94,76 & 33,52 \\
2014 & 83,89 & 97,10 & 85,55 & 92,36 & 37,51 \\
2015 & 88,05 & 100,40 & 106,34 & 95,30 & 43,78 \\
2016 & 71,79 & 85,46 & 76,56 & 79,63 & 36,41 \\
\hline & & $P M 10\left(\mu g / m^{3}\right)$ & & \\
\hline 2012 & 28,57 & 40,63 & 31,01 & 36,01 & 17,82 \\
2013 & 30,13 & 41,15 & 30,33 & 35,28 & 21,07 \\
2014 & 36,67 & 40,12 & 30,32 & 38,66 & 25,37 \\
2015 & 39,78 & 42,12 & 41,10 & 41,71 & 26,59 \\
2016 & 35,25 & 40,80 & 34,79 & 36,75 & 22,83 \\
\hline
\end{tabular}




\section{Análisis del origen de TSP y $\mathrm{PM}_{10}$}

La Figura 5, muestra, el gráfico polar bivariado para las concentraciones medias de TSP y $\mathrm{PM}_{10}$, para los sitios en estudio que cuentan con información meteorológica, con sensores de velocidad y dirección del viento. La opción polarPlot, en Openair, se utilizó para graficar esta información. Se observan altas concentraciones de TSP y $\mathrm{PM}_{10}$ en condiciones de viento mayores a $2 \mathrm{~m} / \mathrm{s}$, con viento predominante desde el NEE, NNE, NE y E, para las estaciones de PV, BR y LC, respectivamente.

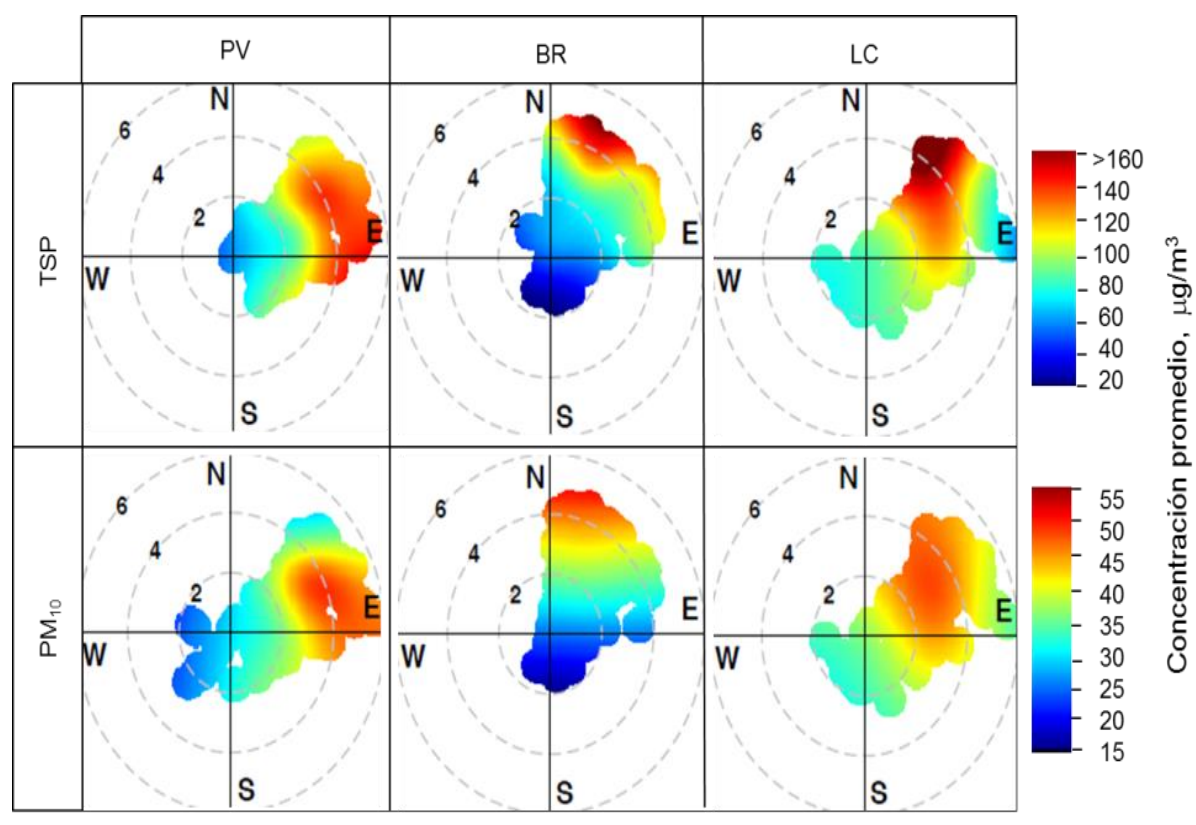

Fig. 5: Diagrama polar bivariante de las concentraciones de $\mathrm{PM}_{10}$ y $\mathrm{TSP}\left(\mu \mathrm{g} / \mathrm{m}^{3}\right)$, en la mina de El Cerrejón en el período 2012 - 2016 (BR: Barrancas, LC: Las Casitas; PT: Patilla).

La estación LC, que registro las valores más alto de TSP y $\mathrm{PM}_{10}$, muestra aporte desde las direcciones NNE, NE y E, entre velocidades del viento 2 a $5 \mathrm{~m} / \mathrm{s}$, para los dos contaminantes. Los máximos promedios se observan en la dirección NE. Este aporte se relaciona, en gran porcentaje, con las actividades mineras de los pits cercanos: PATp, 100p y OREp. Por lo general, estas actividades de emisión de material particulado en los pit, se relaciona con: vías de acarreos, cargue de carbón y material estéril y las emisiones desde tubo de escape de los vehículos. Cuando la dirección del viento proviene del E, se presentan menores promedios del material particulado. En esta dirección, el aporte se relaciona, solo con las actividades del tajo COMp.

Los rangos de velocidad del viento indican aporte de fuente local. La estación BR, muestra aporte desde las direcciones NNE y NE, entre velocidades del viento 4 a $5 \mathrm{~m} / \mathrm{s}$, para los dos contaminantes. Este aporte se relaciona directamente, con las actividades mineras del pit PATp, ubicado al nornordeste de la estación y aporte menor de pit 100p, ubicado al noreste de la estación. La estación PV, muestra aporte desde las direcciones NE y E, entre velocidades del viento 2 a $5 \mathrm{~m} / \mathrm{s}$, para los dos contaminantes. Este aporte se relaciona directamente, con las actividades mineras del pit PATp, ubicado al noreste de la estación y aporte menor de pit COMp, ubicado al este de la estación. La Figura 5, muestra un comportamiento coherente, entre los niveles máximos de los promedios de TSP y $\mathrm{PM}_{10}$ y la ubicación de los pits de operación. También se puede observar en la Figura 5, aportes de fuentes regionales las estaciones BR, LC y PT (velocidad de viento entre 5 y $6 \mathrm{~m} / \mathrm{s}$ ).

La Figura 6, muestra el gráfico polar bivariado, para las concentraciones medias de TSP y $\mathrm{PM}_{10}$, para los sitios en estudio, donde no hay sensores y la información meteorológica de velocidad y dirección del viento, fueron estimadas con el modelo CALMET. Se observaron altas concentraciones de TSP y PM10, en condiciones de viento mayores a $2 \mathrm{~m} / \mathrm{s}$ para la estación PT. Esto indica, al igual que en otras estaciones, que las emisiones, son desde fuentes cercanas. La estación SS registra bajas concentraciones de TSP y $\mathrm{PM}_{10}$, cuando se registran vientos del NE mayores a $3 \mathrm{~m} / \mathrm{s}$, lo cual sugiere una influencia significativa de fuentes regionales viento arriba, de la actividad minera. La estación PT está influenciada por las actividades mineras de los tajos LPUp y TABp (ver Figura 1).

A las condiciones de vientos estimadas en esta estación, también hay alta probabilidad que el aporte de TSP y $\mathrm{PM}_{10}$ se origine, en los tajos ubicados más al norte de la mina. Investigaciones, han demostrado que 
las partículas tienen la mayor concentración cuando están cerca de las fuentes. Para el caso específico de las minas a cielo abierto, la de mayor impacto son las comunidades adyacentes a la explotación (Aneja et al., 2012; Chaulya, 2004). Un estudio aplicando modelo receptor para determinar aportes por fuentes, complementaría los resultados y permitiría confirmar la procedencia del material particulado.

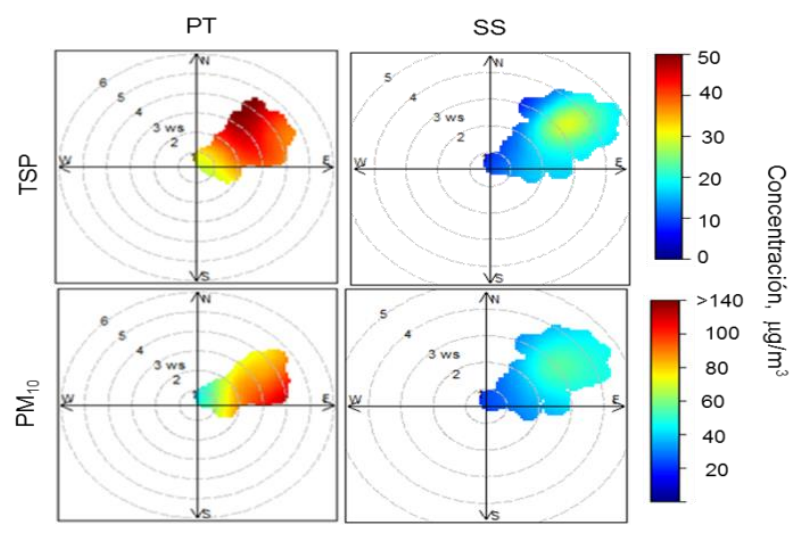

Fig. 6: Diagrama polar bivariante de las concentraciones de PM10 y TSP $\left(\mu \mathrm{g} / \mathrm{m}^{3}\right)$ en la mina de El Cerrejón en el período 2012 - 2016 (PT: Tatilla, SS: Sol y sombra).

\section{CONCLUSIONES}

No se registraron tendencias estadísticamente significativas en los parámetros meteorológicos. Las concentraciones de TSP y $\mathrm{PM}_{10}$ varían a lo largo del año. Los valores promedio más altos se obtuvieron durante la temporada seca. El comportamiento entre los picos de TSP y $\mathrm{PM}_{10}$, indica que las emisiones de polvo son de fuentes similares y locales. Tres de las cinco estaciones, mostraron tendencias de aumento en las concentraciones de $\mathrm{PM}_{10}$.

Solo se observó correlación significativa entre la velocidad del viento y las concentraciones de TSP y PM10. Para el material particulado $\mathrm{PM}_{10}$ no fueron superados los niveles diario y anual de la norma colombiana. Todos los promedios de $\mathrm{PM}_{10}$ en las estaciones BR, LC, PT, PV, superan el nivel anual de la guía de la Organización Mundial de la Salud. Los aportes de material particulado TSP y PM10, se relaciona con las actividades mineras desarrolladas en los pit de explotación, mostrando un comportamiento coherente, entre los niveles máximos de los promedios de TSP y $\mathrm{PM}_{10}$ y la ubicación de los pits de operación. Se registran aportes significativos de $\mathrm{PM}_{10}$ de fuentes regionales, no asociadas a la explotación de carbón.

\section{AGRADECIMIENTOS}

Los autores agradecen al convenio Colciencias-Cerrejón, 1115-524-30465, por el financiamiento del proyecto. También agradecemos el apoyo logístico de la Universidad de Antioquia, la Universidad de La Guajira y la Universidad popular del Cesar. Los donantes no tuvieron ningún papel en el diseño del estudio, la recolección y análisis de datos, la decisión de publicar o la preparación del manuscrito.

\section{REFERENCIAS}

Aneja, V.P., A. Isherwood y P. Morgan, Characterization of particulate matter (PM10) related to surface coal mining operations in Appalachia, Atmospheric Environment, 54, 496-501 (2012)

Argumedo, C.D. y J. Castillo, Chemical characterization of particulated atmospheric matter PM10 in Guajira, Colombia, Revista Colombiana de Química, 45(2), 19-29 (2016)

Bert Brunekreef, S.T.H., Air pollution and health, doi: 10.1016/s0140-6736(02)11274-8, The Lancet, 360 (7002), 12331242 (2002)

BP Statistical Review of World Energy June 2017, 66 ${ }^{\text {th }}$ Edn. BP p.I.c., London (2016)

Carslaw, D., The openair manual open-source tools for analyzing air pollution data, London (2015)

Carslaw, D.C. y K. Ropkins, Openair - An r package for air quality data analysis, Environmental Modelling and Software, 27-28, 52-61 (2012)

Carslaw, D.C. y S.D. Beevers, Characterising and understanding emission sources using bivariate polar plots and k-means clustering, http://doi.org/10.1016/j.envsoft.2012.09.005, Environmental Modelling and Software, 40, 325-329 (2013) 
Castro, H. y N. Rojas, Caracterización físico-química del material particulado y aplicación de modelos receptor en una población cercana a un proyecto minero en el departamento del Cesar, V CASAP y II Cmas South American Conference, Bucaramanga, Pontificia Universidad Bolivariana, 238-247 (2015)

Cerrejón. Proyecto de Expansión liwo'uyaa, Bogota (2017)

Chaulya, S.K., Assessment and management of air quality for an opencast coal mining area, Journal of Environmental Management, 70(1), 1-14 (2004)

$\mathrm{CIOH}$, Climatología de los principales puertos del Caribe Colombiano, Riohacha, Cartagena de Indias (2010)

EEA, Suspended particulates (TSP/SPM), European Environment Agency, Dinamarca, 1-12 (2008)

Ghose, M.K. y S. R. Majee, Assessment of dust generation due to opencast coal mining - an indian case study, Environmental Monitoring and Assessment, 61, 255-263 (2000)

Grange, S.K., A.C. Lewis y D.C. Carslaw, Source apportionment advances using polar plots of bivariate correlation and regression statistics, Atmospheric Environment, 145, 128-134 (2016)

Grange, S.K., Technical note: Averaging wind speeds and directions Technical note, http://doi.org/10.13140/RG.2.1.3349.2006, New Zealand (2014)

Hendryx, M., The public health impacts of surface coal mining, The Extractive Industries and Society, 2, 820-826 (2015)

Hendryx, M. y K.J. Zullig, Higher coronary heart disease and heart attack morbidity in Appalachian coal mining regions, Preventive Medicine, 49(5), 355-9 (2009)

Hopke, P.K., The Application of Receptor Modeling to Air Quality Data, Pollution Atmospherique, $91-109$ (2010)

Huertas, J., M. Huertas, G. Cervantes y J. Diaz, Assessment of the natural sources of particulate matter on the opencast mines air quality, Science of the Total Environment, 493(2000), 1047-1055 (2014)

IDEAM., Informe del Estado de la Calidad del Aire en Colombia 2011-2015, Instituto de Hidrología, Meteorología y Estudios Ambientales, Bogotá D.C., 1-179 (2016)

MADS, Ministerio de Medio Ambiente y Desarrollo Sostenible, Resolución 2254 de 1 noviembre de 2017, Colombia, Norma de Calidad de Aire, 1-11 (2017)

Mesa, O., G. Poveda y otros cinco autores, Distribución espacial y ciclos anual y semianual de la precipitación en Colombia, XIV seminario nacional de hidráulica e hidrología distribución. Bogota D.C., 1-9 (2000)

Monroy, Á.C., S.C. Castillo y otros cinco autores, Simulación y evaluación del impacto de estrategias en el desarrollo del sector minero 2014 -2032, Bogotá D.C.,128 (2014)

Mueller, G.S., A.L. Clayton y otros cinco autores, Geospatial analysis of Cancer risk and residential proximity to coal mines in Illinois, Ecotoxicology and Environmental Safety, 120, 155-162 (2015)

Pabón-Caicedo, J. D. y J. A. Eslava-Ramírez, Generalidades de la distribución espacial y temporal de la temperatura del aire y de la precipitación en Colombia, Meteorología Colombiana, 4, 47-59 (2001)

Pope, C.A. y D. W. Dockery, Health Effects of Fine Particulate Air Pollution: Lines that Connect, Air \& Waste Manage, Association, 56(6), 709-742 (2006)

Ramírez, J.A. y J.I. Del Valle, Local and global climate signals from tree rings of Parkinsonia praecox in La Guajira, Colombia, International Journal of Climatology, 32(7), 1077-1088 (2012)

Rodríguez, J. L., L.C., Angulo, G.M., Restrepo y R.E. Rojano, Evaluation of the models CALMET and CALPUFF in two sites topographically different in the coal mining zone of department of Cesar, Colombia, ProScience, 1, 94-99 (2014)

Rojano, R., H. Arregocés, L. Angulo y G. Restrepo, PM10 emissions due to storage in coal piles in a mining industrial area, Transactions on Ecology and The Environment, 207, 87-97 (2016)

Rojano, R.E., C.A., Manzano y otros cuatro autores, Potential local and regional impacts of particulate matter emitted from one of the world's largest open-pit coal mines, Air Quality, Atmosphere \& Health, 1-10 (2018)

Scire, J.S., D.G, Strimaitis y R.J. Yamartino, Guide for the CALPUFF Dispersion Model, Eearth Tech. Inc. (2000)

Shi, L., A. Zanobetti y otros cinco autores, Low-Concentration PM and Mortality: Estimating Acute and Chronic Effects in a Population-Based Study, Environmental Health Perspectives, 124(1), 45-62 (2016)

Unidad de Planeación Minero energética, UPME, Producción Oficial de Minerales en Colombia (2018)

USEPA, Air emissions (2018)

Watson, J. G. y J. C. Chow, Receptor Models and Measurements for Identifying and Quantifying Air Pollution Sources. In

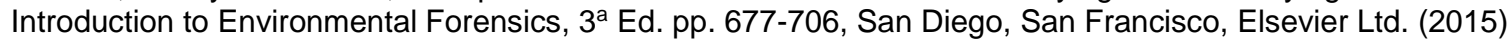

Watson, J.G., J.C. Chow y T.G. Pace, Fugitive dust emissions: Air Pollution Engineering Manual, W. T. Davis (Ed.), pp. 117-135. New York: John Wiley \& Sons (2000) 
